\title{
A randomised controlled trial to assess the relative benefits of large volume spacers and nebulisers to treat acute asthma in hospital
}

\author{
A L Dewar, A Stewart, J J Cogswell, G J Connett
}

\begin{abstract}
Objectives-To compare the clinical effectiveness, acceptability, and cost benefit of administering $\beta_{2}$ agonists by means of a metered dose inhaler and large volume spacer with conventional nebulisers to children admitted to hospital with acute asthma.

Methods-A randomised controlled trial was conducted over five months. Sixty one children older than 3 years admitted to a large teaching hospital and a district general hospital with acute asthma completed the study. Children received either $5 \mathrm{mg}$ of salbutamol up to one hourly by jet nebuliser, or up to 10 puffs of salbutamol $100 \mu \mathrm{g}$ by means of a metered dose inhaler and spacer up to one hourly.

Results-Median hospital stay was 40 hours in the nebuliser group and 36.5 hours in the spacer group. Asthma disability scores at two weeks after discharge were significantly improved in the spacer group. Drug costs were $£ 14.62$ less for each patient in the spacer group.

Conclusions-Large volume spacers are an acceptable, cost effective alternative to nebulisers in treating children admitted with acute asthma, provided that the children can use the mouthpiece, and symptoms are not severe. Their use facilitates effective home treatment by parents, with subsequent reduction in morbidity and re-admission rates.
\end{abstract}

(Arch Dis Child 1999;80:421-423)

Southampton General Hospital, Tremona Road, Southampton SO16 6YD, UK

A L Dewar

G J Connett

Southampton

University Medical

School, Bassett

Crescent East,

Southampton

SO16 7NW, UK

A Stewart

Poole General Hospital, Longfleet

Road, Poole, Dorset BH15 2JB, UK

J J Cogswell

Correspondence to: Dr Connett.

Accepted 12 January 1999
Keywords: acute asthma; large volume spacer; metered dose inhaler; nebuliser; $\beta_{2}$ agonists; cost effectiveness

In recent years, the treatment of childhood asthma has been an increasingly demanding and expensive health care problem. It has been estimated that hospital admissions for asthma in the UK in 1988 cost $£ 57.6$ million. ${ }^{1}$ The optimal management of asthma has important implications, not just financially, but also for individual children's social and personal development.

Spacer devices have become an increasingly popular method of delivering inhaled drugs to treat childhood asthma. Using a large volume spacer successfully overcomes the technical problems associated with using metered dose inhalers alone, as long as the child is able to breathe in and out through the mouthpiece. ${ }^{2} 3$

Several recent trials have shown that $\beta_{2}$ agonists can be delivered by means of a metered dose inhaler and spacer as an acceptable and effective alternative to nebulised drug delivery to treat acute childhood asthma. ${ }^{4-11}$ These two methods of drug delivery have been compared recently in a large meta-analysis of their use in emergency departments. ${ }^{12}$ Results showed that clinical outcomes were similar and that side effects were less problematical using a metered dose inhaler and spacer. The largest study, performed by Chou and colleagues, suggested that the use of spacers enabled shorter treatment times and earlier discharge from the emergency department. ${ }^{4}$ These benefits were more apparent in children.

Despite these data, nebulisers continue to be the mainstay for treatment of acute asthma in hospital. We have postulated that the increasing awareness that nebulisers are effective in acute asthma has produced a population of families who repeatedly seek inpatient access to this favoured form of treatment. ${ }^{13}$ The aim of our study was to discover whether metered dose inhaler and spacer treatment could effectively replace nebulised bronchodilators for children admitted to paediatric hospital wards. We hoped that by teaching families to administer these treatments immediately after admission they might be better able to continue treatment at home. By using the inpatient stay as an opportunity to educate the parent and child in self treatment from the beginning of the admission, we hoped that this would also enable them to treat subsequent attacks without needing readmission.

\section{Patients and methods}

Our study was carried out at Southampton University Hospital and Poole District General Hospital. Together, these hospitals serve a paediatric population of 180000 . We invited all children aged 3 years or more who required hospital admission for acute asthma to participate. Exclusion criteria included children unable to use a spacer mouthpiece effectively, children with severe symptoms requiring intravenous treatments, and children readmitted during the five month study period. Informed consent was obtained from parents before treatment. We carried out randomisation using sequential presealed envelopes, which randomly allocated children into each of the two study groups.

Children in the control (nebuliser) group received salbutamol $5 \mathrm{mg}$ up to one hourly by jet nebuliser driven by oxygen at $6-8 \mathrm{l} / \mathrm{min}$. All children also received oral prednisolone at $2 \mathrm{mg} / \mathrm{kg}$ (maximum dose, $60 \mathrm{mg}$ ) on admission 
Table 1 Clinical characteristics of the two groups at admission and discharge

\begin{tabular}{|c|c|c|}
\hline & Nebuliser group $(n=33)$ & Spacer group $(n=29)$ \\
\hline \multicolumn{3}{|c|}{ Heart rate (beats/minute) } \\
\hline On admission & $129.8(122.9$ to 136.6$)$ & 124.2 (119.6to128.8) \\
\hline At discharge & 99.3 (94.1 to 104.6$)$ & 101.2 (96.6 to 105.8$)$ \\
\hline \multicolumn{3}{|c|}{ Respiratory rate (breaths/minute) } \\
\hline On admission & 39.7 (36.1 to 43.4$)$ & 36.4 (34.2 to38.7) \\
\hline At discharge & $27.2(25.4$ to 29.0$)$ & $26.6(24.7$ to 28.6$)$ \\
\hline \multicolumn{3}{|c|}{ Oxygen saturation $(\%)$} \\
\hline On admission & 91.9 (90.7 to 93.0$)$ & 93.2 (92.4 to 93.9$)$ \\
\hline At discharge & 96.1 (95.9 to 96.5$)$ & 96.4 (95.9 to 96.9$)$ \\
\hline \multicolumn{3}{|l|}{ Temperature $\left({ }^{\circ} \mathrm{C}\right)$} \\
\hline On admission & $36.6(36.7$ to37.1) & $36.9(36.8$ to 37.3$)$ \\
\hline At discharge & $36.6(36.5$ to 36.8$)$ & 36.6 (36.5 to 36.7$)$ \\
\hline
\end{tabular}

Values are mean (95\% confidence intervals).

and repeated on subsequent mornings for three to five doses according to their recovery.

Children in the study (spacer) group received salbutamol $100 \mu \mathrm{g}$ by large volume spacer (volumatic), up to 10 puffs one hourly. All children in this group received oral prednisolone according to the same ward protocol as the control group. We administered oxygen by face mask or nasal prongs in children who had oxygen saturations measuring $<93 \%$ after bronchodilator treatment. Children and parents in the spacer group were instructed and supervised on the optimal use of the delivery device. This instruction was carried out by the ward nursing staff, who had themselves been taught about optimal inhaler techniques by the respiratory paediatricians (GC and AD). In both groups, the frequency of bronchodilator usage and the use of other medications was at the discretion of the attending on call paediatrician. The on call paediatric team also determinded the time of discharge for all children in our study. The only other intervention was to provide the families of children in the spacer group with a written treatment plan for managing acute asthma. We gave all families strict instructions to give bronchodilators no more often than four hourly and to return to hospital if relief medication was needed more frequently. Instructions included recourse to the use of a short course of oral steroids if symptoms were poorly responsive to 10 puffs of salbutamol four hourly.

Two weeks after discharge, all families were contacted by telephone and a measurement of asthma morbidity over the two week period was calculated using the "index of perceived symptoms" questionnaire developed by Usherwood et al. ${ }^{14}$

\section{STATISTICS}

We projected the target sample size using estimations from asthma admission data over the previous two years. We estimated 100 asthma admissions to represent a reasonable number expected over our study period. We did not complete the main comparative analyses on an intention to treat basis because it was decided to exclude those children with very severe asthma who required intravenous treatment. We performed analyses on an IBM compatible PC using SPSS for windows. We compared parametric data by means of the independent $t$ test and non-parametric data by means of the Mann-Whitney U test.
Table 2 Demographic data for nebuliser and spacer groups

\begin{tabular}{lll}
\hline & Nebuliser & Spacer \\
\hline Number of patients & 33 & 29 \\
Sex (male:female) & $24: 9$ & $16: 13$ \\
Mean age (years) & 8.0 & 6.9 \\
Use of prophylactic inhaled steroids & 19 & 13 \\
Family history of asthma & 16 & 18 \\
Family history of atopy & 13 & 9 \\
Parental smoking & 11 & 9 \\
Cat or dog at home & 18 & 15 \\
\hline
\end{tabular}

The Usherwood index of perceived symptoms asthma morbidity questionnaire generated scores for daytime disturbance, night-time disturbance, and asthma disability with fixed responses to nine questions scoring 1 for no symptoms and 5 for maximum symptoms.

\section{Results}

Over the five month study period a total of 78 eligible children were admitted to the two hospitals. Two children required immediate intravenous treatment and were excluded before randomisation. Five children were withdrawn because of subsequent deteriorations requiring intravenous intervention (three in the spacer group and two in the nebuliser group). Nine children who were readmitted during the five month study period were not restudied. Thirty three children completed the study in the nebuliser group (mean age, 8.0 years) and 29 in the spacer group (mean age, 6.9 years). Surprisingly, no child in the study was too obstructed to operate the spacer valve, although this was not attempted in the two children requiring immediate intravenous treatment.

Statistical analyses showed no significant difference in clinical characteristics and baseline severity between the two groups on admission. Both groups were similarly improved at discharge (table 1). Demographic data was also equally balanced between the two groups, apart from a non-significant predominance of boys in the nebuliser group (table 2).

We could not use mean values for length of hospital stay because a small number of lengthy inpatient stays caused skewing of the data. When we compared median values, the length of hospital stay was 40.0 hours for the nebuliser group and 36.5 hours for the spacer group. This difference was not significant, but box plot and interquartile ranges suggest a trend towards shorter admission times for the spacer group (fig 1).

We calculated the cost of drug treatment for each individual child. In the nebuliser group this consisted of the fixed cost for each patient

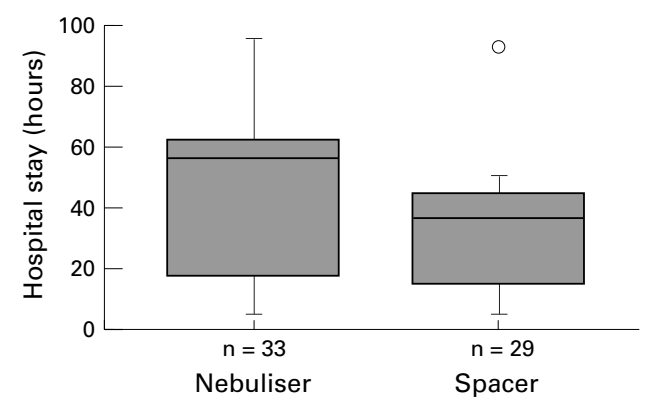

Figure 1 Box plots showing hospital stay times for both groups. 
Table 3 Asthma morbidity scores for the two groups two weeks after discharge

\begin{tabular}{llclc}
\hline & Score range & $\begin{array}{l}\text { Nebuliser group } \\
\text { median (range) }\end{array}$ & $\begin{array}{l}\text { Spacer group } \\
\text { median (range) }\end{array}$ & p Value \\
\hline Daytime score & 4 to 20 & $10(4-17)$ & $6(4-14)$ & 0.001 \\
Night time score & 2 to 20 & $4(2-10)$ & $3(2-6)$ & $<0.05$ \\
Disability score & 5 to 25 & $8(5-19)$ & $6(5-16)$ & $>0.05$ \\
\hline
\end{tabular}

of disposable nebuliser equipment, and variable costs of salbutamol nebules and prednisolone. The costs for the spacer group consisted of the fixed price of a metered dose inhaler and volumatic spacer for each patient, together with prednisolone used as an inpatient, and the additional course of prednisolone for future use at home. We did not include the cost of oxygen. The mean cost in the nebuliser group was $£ 20.25 /$ patient, and in the spacer group $£ 5.43 /$ patient. The difference was highly significant (independent sample $t$ test, $\mathrm{p}<0.001)$.

We obtained an assessment of asthma morbidity scores at two weeks after discharge from 29 of 33 patients in the nebuliser group and 28 of 29 patients in the spacer group. Scores favoured the spacer group (table 3 ).

There were nine readmissions during the study period. One child had been excluded previously because of the need for intravenous treatment. Of the remainder, six were in the nebuliser group and two in the spacer group $(\mathrm{p}>0.05)$. This trend favouring the spacer group persisted over a 12 month follow up period with readmission rates of $27.2 \%$ for the nebuliser group and $13.8 \%$ for the spacer group. One child in each group was readmitted on more than one occasion.

\section{Discussion}

Despite convincing evidence for the effectiveness of $\beta_{2}$ agonist treatment using metered dose inhalers with spacers, ${ }^{10}$ there remains a reluctance to use these devices as first line treatment during an acute attack. Nebulisers continue to be the preferred method of treatment. ${ }^{15-18}$ Our data were collected from acutely ill hospitalised children and are consistent with studies reporting the advantages of metered dose inhalers and spacers over nebulisers in the emergency room, ${ }^{10}$ and therefore question the current dogma.

Surprisingly, all children over the age of 3 years and who satisfied entry criteria for our study, were able to use spacers via the mouthpiece without needing a face mask attachment. Subsequently, however, we have encountered some 3 year old children who have had problems with this delivery system.

Within a cash limited health service there are increasing pressures to explore the use of more cost effective treatments. If a district general hospital admitting 200 children with asthma each year treated all such admissions with spacers, the saving on drug expenditure would be $\sim £ 3000$. This saving could be increased if families were encouraged to bring their own spacer devices into hospital during admissions. The similar recovery during hospital stay in those administered bronchodilators via a spacer or nebuliser clearly demonstrates the effectiveness of the cheaper spacer.

The children in the spacer group demonstrated a consistent and significant improvement in symptoms at two weeks, compared with those in the nebuliser group. We suggest that this difference is the result of continuous inpatient training in the use of the metered dose inhaler and spacer during an acute attack. Enabling nursing staff to teach families about the use of metered dose inhalers and spacers led to effective treatment on the paediatric wards and increased the confidence of families to continue similar treatment at home. This "training" might also have been a factor in reducing admission rates over the next 12 months, although the written information about managing future attacks was probably also important in achieving this.

Reversing the "nebuliser culture" in the two hospitals involved in our study has been a slow and difficult process. Despite this, the initial reluctance among nursing staff to change a long established practice has gradually been overcome and both units are choosing to use metered dose inhalers and spacers as first line treatment for hospital admissions.

Further studies involving larger numbers of patients might help confirm our findings and lead to the more widespread use of this cost effective option for asthma management.

1 Clark TJH. The occurrence and cost of asthma. Worthing: Cambridge Medical Publications, 1990

2 Barry PW, O'Callaghan C. Inhalational drug delivery from seven different spacer devices. Thorax 1996;51:835-40.

3 O'Callaghan C, Barry P. Spacer devices in the treatment of asthma. BMF 1997;314:1061-2.

4 Chou KJ, Cunningham SJ, Crain EF. Metered dose inhalers with spacers vs nebulisers for paediatric asthma. Arch Paediatr Adolesc Med 1995;149:201-5.

$5 \mathrm{Lin}$ YZ, Hsieh KH. Metered dose inhaler and nebuliser in acute asthma. Arch Dis Child 1995;72:214-18.

6 Parkin PC, Saunders NR, Diamond SA, Winders PM, Macarthur C. Randomised trial spacer v nebuliser for acute asthma. Arch Dis Child 1995;72:239-40.

7 Kerem E, Levison H, Schuh S, et al. Efficacy of albuterol administered by nebuliser versus spacer device in children with acute asthma. F Pediatr 1993;123:313-17.

8 Pendergast J, Hopkins J, Timms B, Van Asperen PP. Comparative efficacy of terbutaline administered by nebuhaler and by nebuliser in young children with acute asthma. Med 7 Aust 1989;151:406-8.

9 Benton G, Thomas C, Nickerson B, McQuitty JC, Okikawa J. Experience with metered dose inhaler with a spacer in a paediatric emergency department. Am f Dis Child 1989; 143:678-81.

10 Fuglsang G, Pederson S. Comparison of nebuhaler and nebuliser treatment of acute severe asthma in children. Eur f Respir Dis 1986;69:109-13.

11 Freelander M, Van Asperen PP. Nebuhaler versus nebuliser in children with acute asthma. BMF 1984;288:1872-4.

12 Cates CJ. Comparison of holding chambers and nebulisers for beta-agonists in acute asthma. A systematic review of randomised control trials. (Cochrane library). Update Software, 1997 (updated quarterly).

13 Connett GJ, Warde C, Wooler E, Lenney W. Audit strategies to reduce hospital admissions for acute asthma. Arch Dis Child 1993;69:202-5.

14 Usherwood TP, Scrimgeour A, Barber JH. Questionnaire to measure perceived symptoms and disability in asthma. Arch Dis Child 1990;65:779-81.

15 British Thoracic Society, British Paediatric Association, Royal College of Physicians of London, The King's Fund Centre, National Asthma Campaign, et al. Guidelines on the management of asthma. Thorax 1993;48(suppl):S1-24.

16 Blake KV, Hoppe M, Harman E, Hendalls L. Relative amounts of albuterol delivered to lung receptors from a metered dose inhaler and nebuliser solution. Chest 1992;101:309-13.

17 Canny GJ, Reiman J, Healy R. Acute asthma: observations regarding the management of a paediatric emergency room. Paediatrics 1989;83:507-12.

18 Pederson S. Inhalers and nebulisers. Which to choose and why. Respir Med 1996;90:69-77. 